\title{
EQUILATERAL SETS IN MINKOWSKI SPACES
}

\author{
C. M. PETTY
}

Abstract. The structure of equilateral sets in an $n$-dimensional Minkowski space $M^{n}$ is shown to be closely related to the properties of antipodal sets. The range of the cardinality of maximal equilateral sets in $M^{n}$ is obtained and a subset characterization of antipodal sets is derived and applied to equilateral sets.

1. Introduction. A subset $S$ of a metric space is said to be equilateral provided each pair of points in $S$ have the same distance. The structure of such sets is well known in euclidean, hyperbolic and spherical spaces but, due to the metric peculiarities, little is known about such sets in elliptic $n$-space for $n>5$. See Blumenthal [1], Blumenthal and Kelly [2], Haantjes [6], and van Lint and Seidel [8].

We study here the structure of such sets in (real) finite dimensional Banach spaces (Minkowski spaces) of dimension $n>0$. We adopt the terminology and notation in DGK [4].

2. Equilateral sets. A subset $S$ of an $n$-dimensional real linear space $R^{n}$ is said to be antipodal provided for each pair of points $p, q \in S$ there exist disjoint parallel support hyperplanes $P, Q$ such that $p \in P$, $q \in Q$. Properties of antipodal sets have been studied in [3] and [5]. An antipodal set is finite and $S$ is the set of vertices of the antipodal polytope $A=$ conv $S$.

A line $L$ in a Minkowski space $M^{n}$ is normal to a hyperplane $H$ (or $H$ is transversal to $L$ ) at a point $f$ if $L \cap H=\{f\}$ and the distance $x f \leqq x y$ for $x \in L$ and $y \in H$. The line through two points $p$ and $q$ will be denoted by $L(p, q)$.

THEOREM 1. Let $S$ be an equilateral set in a Minkowski space $M^{n}$. Then $S$ is an antipodal set such that for every pair of points $p, q \in S$ the hyperplanes through $p$ and $q$ transversal to $L(p, q)$ are support hyperplanes to $S$.

Proof. Let diam $S=d>0$ and put $S(z ; \rho)=\{x:\|x-z\|=\rho\}$. If $p, q \in S, p \neq q$, then the sphere $S(p ; d) \supset S \sim p$. Let $Q$ be a support hyperplane to $S(p ; d)$ at $q$. Then the translate $S(p ; d)+(q-p)$ $=S(q ; d)$ has a support hyperplane $P$ at $p$ parallel to $Q$ and, since $S(q ; d) \supset S \sim q, P$ is a support hyperplane of $S$.

Received by the editors August 1, 1969.

AMS 1970 subject classifications. Primary 52A20, 52A50; Secondary 52A25.

Key words and phrases. Equilateral sets, Minkowski spaces, antipodal sets. 
THEOREM 2. Let $S$ be an antipodal set in $R^{n}$. Then there exists a norm on $R^{n}$ such that $S$ is an equilateral set.

Proof. Let $A=$ conv $S$. Since a norm on a subspace of $R^{n}$ can be extended to a norm on $R^{n}$, we may assume that $\operatorname{dim} A=n$. The $\operatorname{dif}$ ference body $A-A$ is a convex body which is centrally symmetric about the origin $O$. Since $S$ is antipodal, for $p, q \in S, p \neq q, p-q$ is a boundary point of $A-A$. Consequently, $A-A$ determines a norm on $R^{n}$ such that $A-A$ is the unit cell and $S$ is an equilateral set with $\operatorname{diam} S=1$ which completes the proof.

If an antipodal set $S$ has the property that for each pair of points $p, q \in S$ there exist parallel support hyperplanes $P$ and $Q$ such that $P \cap S=\{p\}, Q \cap S=\{q\}$, then $S$ is said to be strictly antipodal (Grünbaum [5]). From the proof of Theorem 1, it is seen that if a set $S$ consists of more than one point and $(S-S) \sim 0$ are exposed points of $S(0 ; d), d>0$, then $S$ is a strictly antipodal equilateral set of diameter $d$. If the unit cell is strictly convex then every equilateral set is strictly antipodal. We prove next a definitive statement of this situation.

If $p$ is a boundary point of a convex set $K$ then the tangent cone to $K$ with apex $p$, denoted by $C(p ; K)$, is defined to be the closure of the smallest cone with apex $p$ which contains $K$. An antipodal polytope $A$ such that $S=$ vert $A$ is an equilateral set will be called an equilateral polytope. The unit Minkowski cell $B^{n}$ is said to have property $P$ provided there exists a support hyperplane $H$ to $B^{n}$ which contains equilateral polytopes $A_{1}$ and $A_{2}$ of diameter 2 such that $\frac{1}{2}\left(A_{1}+A_{2}\right) \subset H \cap B^{n}$ and there exist $p_{i} \in$ vert $A_{i}(i=1,2)$ such that $C\left(p_{1} ; A_{1}\right)$ contains a ray $R_{1}$ opposite to a ray $R_{2}$ of $C\left(p_{2} ; A_{2}\right)$, i.e. $R_{1}+R_{2}$ is a line.

TheOREM 3. Every equilateral set in $M^{n}$ is strictly antipodal if and only if the unit Minkowski cell $B^{n}$ does not have property $P$.

Proof. Suppose $B^{n}$ has property $P$. Since $R_{i}$ (with apex $p_{i}$ ) must intersect the polytope $A_{i}$ in a segment, $\frac{1}{2}\left(p_{1}+p_{2}\right)$ is not an extreme point of $\frac{1}{2}\left(A_{1}+A_{2}\right)$. Let $S_{i}=\operatorname{vert} A_{i}, S=\left(\frac{1}{2} S_{1}\right) \cup\left(-\frac{1}{2} S_{2}\right)$ and $A=\operatorname{conv} S$. Then property $P$ implies that $S$ is an equilateral set of diameter 1 . Now $S$ is strictly antipodal if and only if for any $x, y \in S, x \neq y, x-y$ is an extreme point (vertex) of $A-A$. But $A-A \supset \frac{1}{2}\left(A_{1}+A_{2}\right)$ and $\frac{1}{2} p_{1},-\frac{1}{2} p_{2} \in S, \frac{1}{2} p_{1} \neq-\frac{1}{2} p_{2}$. Therefore $S$ is not strictly antipodal.

Now suppose that $S$ is an equilateral set of diameter 2 which is not strictly antipodal and let $A=$ conv $S$. There exist $p, q \in S, p \neq q$, such that $p-q$ is not an extreme point of $A-A$. It follows that $C(p ; A)$ 
contains a ray $R_{1}$ with apex $p$ parallel to a ray $\left(-R_{2}\right)$ with apex $q$ and contained in $C(q ; A)$. We may assume that $\frac{1}{2}(p+q)$ is the origin and let $H$ be a support hyperplane to $B^{n}$ at $p$. By Theorem $1, H$ and $-H$ are also support hyperplanes to $A$ and consequently $H$ and $-H$ contain $R_{1}$ and $\left(-R_{2}\right)$ respectively. Let $A_{1}=H \cap A,-A_{2}=(-H)$ $\cap A, p_{1}=p, p_{2}=-q=p_{1}$. Since $A_{1}$ and $-A_{2}$ are faces of $A$ consisting of more than one point, it follows that $A_{i}$ is an equilateral polytope with $\operatorname{diam} A_{i}=2(i=1,2)$. If $x_{i} \in A_{i}$ then, since $x_{1} \in H,-x_{2} \in-H$ and $\operatorname{diam} A=2$, we have $\left\|x_{1}+x_{2}\right\|=2$. Consequently, $\frac{1}{2}\left(A_{1}+A_{2}\right)$ $\subset H \cap B^{n}$ and $B^{n}$ has property $P$ which completes the proof.

If some 2-dimensional central section of $B^{n}$ is a parallelogram then $B^{n}$ has property $P$. However, the converse is not true in general for $n>2$. In $R^{3}$, let

$$
S=\{(0,0,0),(1,0,0),(0,1,0),(0,0,1),(\sqrt{ } 3 / 3, \sqrt{ } 3 / 3,1)\} .
$$

The five points in $S$ form an antipodal set which is not strictly antipodal. The 20 points in $(S-S) \sim 0$ lie in 3 parallel planes. Five of the six points in $x_{3}=1\left(x_{3}=-1\right)$ are the vertices $V_{1}\left(V_{2}\right)$ of a convex pentagon and the sixth point, which lies on the $x_{3}$ axis, is in the relative interior of the pentagon. The remaining 8 points lie on the ellipse $E: x_{1}^{2}+x_{1} x_{2}+x_{2}^{2}=1, x_{3}=0$. Let $B^{3}=\operatorname{conv}\left(V_{1} \cup V_{2} \cup E\right)$. The elliptic cylinder $x_{1}^{2}+x_{1} x_{2}+x_{2}^{2}=1$ contains $V_{1} \cup V_{2}$ with the six points $(0, \mp 1$, $\pm 1),(\mp 1,0, \pm 1),( \pm \sqrt{ } 3 / 3, \pm \sqrt{ } 3 / 3, \pm 1)$ lying on the cylinder. Since any segment on this cylinder lies on a generator, the extreme points of $B^{3}$ constitute the set $V_{1} \cup V_{2} \cup E$. In the Minkowski space with $B^{3}$ as unit cell the set $S$ is equilateral with diameter 1 and consequently $B^{3}$ has property $P$. Suppose some central section of $B^{3}$ is a parallelogram. Since the points in $E$ are extreme points of $B^{3}$, a pair of opposite vertices of the parallelogram belong to $E$. By considering parallel support planes to $B^{3}$ at the midpoints of opposite sides of the parallelogram, one sees that some projection of $B^{3}$ is a parallelogram $Q$. Now the projection of $E$ is either an ellipse or a segment and consequently $E$ projects onto a diagonal of $Q$. But then the pentagons would project on to segments parallel to this diagonal blocking off the other two vertices of $G$. Therefore, no central section of $B^{3}$ can be a parallelogram.

An equilateral set is said to be maximal if it is not a proper subset of any other equilateral set.

THEOREM 4. If $S$ is a maximal equilateral set in $M^{n}$ then

$$
\min (4, n+1) \leqq \operatorname{card} S \leqq 2^{n},
$$


where the bounds are sharp for all $n \geqq 1$. The right-hand equality holds if and only if $S$ is the set of vertices of a parallelotope $A=\operatorname{conv} S$ and $A$ is a Minkowski cell.

Proof. Danzer and Grünbaum [3] have shown that the maximum number of points in an antipodal set in $R^{n}$ is $2^{n}$ and this maximum number is attained only if the points of the antipodal set are the vertices of an $n$-dimensional parallelotope. Suppose then that card $S$ $=2^{n}$, $\operatorname{diam} S=d$, and $y$ is the center of the parallelotope $A=\operatorname{conv} S$. The Minkowski cell with center $y$ and radius $d / 2$ contains $S$ on its boundary. A chord of this cell through $y$ and parallel to an edge of $A$ has the same length as the edge and consequently the endpoints of the chord are the centers of opposite $(n-1)$-dimensional faces (facets) of $A$. A supporting plane to this cell at an endpoint of this chord must contain the corresponding facet of $A$ and it follows that $A$ is a Minkowski cell.

For $n=2$, it is easily seen that each pair of points $p, q$ with distance $p q=d>0$ can be extended, in either closed half-space bounded by $L(p, q)$, to at least one equilateral triple with diameter $d$. We will show that each equilateral triple in $M^{3}$ can be extended to an equilateral quadruple.

In $M^{3}$, let $0, p_{1}, p_{2}$ be an equilateral triple of diameter 1 lying in a plane $H$. Let $F=K \cap S(0 ; 1)$, where $K$ is a closed half-space bounded by $H$. Define the continuous mapping $f: S(0 ; 1) \rightarrow E^{2}$ by $f(z)=\left(z p_{1}\right.$, $z p_{2}$ ). Since $F$ and $-F$ are simply connected, $f(F)$ and $f(-F)$ are simply connected. If $C=H \cap S(0 ; 1)$, we will show that $f(C)$ either encircles $(1,1)$ or $(1,1) \in f(C)$. In the latter case, there exists $q \in C$ such that $f(q)=(1,1)$ and $0, p_{1}, p_{2}, q$ are a planar equilateral quadruple which are the vertices of a parallelogram which is also a Minkowski circle. In the former case, $0, p_{1}, p_{2}$ can be extended in either closed half-space $K$ or $-K$ to a three-dimensional equilateral quadruple.

Orient the Minkowski circle $C$ by the shorter of the two arcs from $p_{1}$ to $p_{2}$. As $z$ traverses $C$ in the positive sense from a point $x \in C$ to $-x$, the distance $x z$ is nondecreasing. For if $L$ is a supporting line in $H$ to $C$ at $z$ with orientation induced by that of $C$, then the ray from $x$ parallel to the ray from 0 to $z$ cuts $L$ in a point $f$ which precedes $z$ on $L$ and $f$ is a foot of $x$ on $L$. Let $\left\|p_{1}+p_{2}\right\|=s, 1 \leqq s \leqq 2$. As $z$ traverses $C$ in the positive sense starting from $p_{1}, z$ encounters $p_{1}, p_{2}$, $-p_{1},-p_{2}, p_{1}$ with $f\left(p_{1}\right)=(0,1), f\left(p_{2}\right)=(1,0), f\left(-p_{1}\right)=(2, s), f\left(-p_{2}\right)$ $=(s, 2)$. Following $z$ along the four arcs, one shows that either $f(C)$ encircles $(1,1)$ or $(1,1) \in f(C)$. 
For the general case, the extension of equilateral sets stops with four points. In $R^{n}, n \geqq 3$, let $S^{n-2}$ be the euclidean sphere in the $(n-1)$-dimensional subspace $x_{n}=0$ and let $p_{1}=(0, \cdots, 0,1)$, $p_{2}=(0, \cdots, 0,-1)$. In the Minkowski space with unit cell $\operatorname{conv}\left(S^{n-2} \cup p_{1} \cup p_{2}\right)$, the points $p_{1}, p_{2}, x,-x$ for $x \in S^{n-2}$ form a maximal equilateral quadruple of diameter 2 . This completes the proof.

More restrictive bounds for card $S$ are obtained in Theorem 4 if $B^{n}$ does not have property $P$ (Theorem 3 ). However, the best bounds are unknown for $n>3$. For $n=3$ we obtain $4 \leqq \operatorname{card} S \leqq 5$ (Grünbaum [5]).

TheOREM 5. Let $S$ be a $k$-dimensional subset of $R^{n}, 2 \leqq k \leqq n$. If, for each subset of $S$ with $k+2$ or fewer points there exists a norm on $R^{n}$ such that this subset is equilateral, then there exists a norm on $R^{n}$ such that $S$ is equilateral.

Proof. The proof follows from Theorems 1, 2 and the following result.

Theorem 6. If $S$ is a $k$-dimensional set in $R^{n}, 2 \leqq k \leqq n$, such that every subset of $S$ with $k+2$ or fewer points is antipodal then $S$ is antipodal.

Proof. We may assume that $S$ lies in a $k$-dimensional subset of euclidean space $E^{k}$ with unit sphere $S^{k-1}$ and inner product denoted by $\langle x, y\rangle$. Let $S_{0}$ be a $k$-dimensional finite subset of $S$ with points $p, q, x_{1}, \cdots, x_{m}$. We may assume that $q$ is the origin and $m>k$. Put $K_{i}=\left\{u:|u|=1,\left\langle\left(p-x_{i}\right), u\right\rangle \geqq 0,\left\langle x_{i}, u\right\rangle \geqq 0\right\}, i=1, \cdots, m$. There exist disjoint parallel support hyperplanes to $S_{0}$ at $p$ and $q$ if and only if $K=K_{1} \cap \cdots \cap K_{m}$ is nonempty. By hypothesis, $p-x_{i}, x_{i}$ are not collinear with the origin $q$ and therefore $K_{i}$ is a (closed) homology cell in $S^{k-1}$. Each $K_{i}$ is contained in the hemisphere $\{u:|u|=1$, $\langle p, u\rangle \geqq 0\}$ and, from the hypothesis, each intersection of $k$ or fewer members of the family $\left\{K_{i}\right\}$ is a homology cell. Consequently, by Helly's topological theorem (Helly [7]), $K$ is nonempty (see DGK [4] for spherical analogues of Helly's theorem). Since this argument may be applied to any two points of $S_{0}$ we conclude that $S_{0}$ is antipodal. The set $S$ is therefore finite since $S_{0}$ can have at most $2^{k}$ points and we may set $S=S_{0}$, which completes the proof.

To see that the number $k+2$ cannot be reduced in the hypotheses of Theorems 5 and 6 , one may consider the set $S$ consisting of the vertices of a $k$-simplex and its centroid. 


\section{REFERENCES}

1. L. M. Blumenthal, Theory and applications of distance geometry, Clarendon Press, Oxford, 1953, MR 14, 1009.

2. L. M. Blumenthal and L. M. Kelly, New metric-theoretic properties of elliptic space. Univ. Nac. Tucumán Rev. Ser A7 (1949), 81-107. MR 11, 533; 872.

3. L. Danzer and B. Grünbaum, Über zwei Probleme bezüglich konvexer Körper von P. Erdös und von V. L. Klee, Math. Z. 79 (1962), 95-99. MR 25 \#1488.

4. L. Danzer, B. Grünbaum and V. Klee, Helly's theorem and its relatives, Proc. Sympos. Pure Math., vol. 7, Amer. Math. Soc., Providence, R. I., 1963, pp. 101-180. MR $28 \# 524$.

5. B. Grünbaum, Strictly antipodal sets, Israel J. Math. 1 (1963), 5-10. MR 28 $\# 2480$.

6. J. Haantjes, Equilateral point-sets in elliptic two- and three-dimensional spaces, Nieuw Arch. Wisk. (2) 22 (1948), 355-362. MR 9, 369.

7. E. Helly, Über Systeme abgeschlossener Mengen mit gemeinschaftlichen Punkten, Monatsh. Math. 37 (1930), 281-302.

8. J. H. van Lint and J. J. Seidel, Equilateral point sets in elliptic geometry, Nederl. Akad. Wetensch. Proc. Ser. A 69 = Indag. Math. 28 (1966), 335-348. MR 34 \#685.

University of Missouri-Columbia, Columbia, Missouri 65201 